\section{RSP}

http://www.rsp.fsp.usp.br/
Revista de Saúde Pública

\title{
Fatores associados a sintomas osteomusculares em profissionais que trabalham sentados
}

\author{
Anália Rosário Lopes' (iD, Celita Salmaso Trelha" (iD, Maria Lúcia do Carmo Cruz Robazzi"' \\ Roberta Alvarenga Reis ${ }^{\mathrm{Iv}}$ (ID, Maria José Bistafa Pereirav ${ }^{\mathrm{V}}$ (D), Claudia Benedita dos Santos ${ }^{\mathrm{v}}$ \\ 1 Universidade Federal da Integração Latino-Americana. Instituto Latino-Americano de Ciências da Vida e da \\ Natureza. Curso de Medicina. Foz do Iguaçu, PR, Brasil \\ " Universidade Estadual de Londrina. Departamento de Fisioterapia. Londrina, PR, Brasil \\ III Universidade de São Paulo. Escola de Enfermagem de Ribeirão Preto. Departamento de Enfermagem Geral e \\ Especializada. Ribeirão Preto, SP, Brasil \\ iv Universidade Federal do Rio Grande do Sul. Faculdade de Odontologia. Departamento de Odontologia \\ Preventiva e Social. Porto Alegre, RS, Brasil \\ $\checkmark$ Universidade de Ribeirão Preto Campus Ribeirão. Programa de Mestrado Profissional Educação em Saúde. \\ Ribeirão Preto, SP, Brasil \\ v' Universidade de São Paulo. Escola de Enfermagem de Ribeirão Preto. Departamento de Enfermagem Materno \\ Infantil e Saúde Pública. Ribeirão Preto, SP, Brasil
}

\section{RESUMO}

OBJETIVOS: Estimar a prevalência de sintomas osteomusculares e analisar os fatores a eles associados em profissionais de setores administrativos que trabalham predominantemente na postura sentada.

Correspondência:

Anália Rosário Lopes

Universidade Federal da Integração

Latino-Americana

Av. Tarquínio Joslin dos Santos,

n.1000, Jardim Universitário.

85870-901, Foz do Iguaçu,

PR, Brasil

E-mail: analia.lopes@unila.edu.br

Recebido: 6 mai 2020

Aprovado: 1 jul 2020

Como citar: Lopes AR, Trelha CS, Robazzi MLCC, Reis RA, Pereira MJB, Santos CB. Fatores associados a sintomas osteomusculares em profissionais que trabalham sentados. Rev Saude Publica. 2021;55:2.

https://doi.org/10.11606/s15188787.2021055002617

Copyright: Este é um artigo de acesso aberto distribuído sob os termos da Licença de Atribuição Creative Commons, que permite uso irrestrito, distribuição e reprodução em qualquer meio, desde que o autor e a fonte originais sejam creditados.
MÉTODOS: Trata-se de estudo transversal com dados obtidos de 451 trabalhadores de instituição pública federal na região Sul do país. A variável dependente foi o número de sintomas osteomusculares nos últimos 12 meses, aferido utilizando-se o Questionário Nórdico de Sintomas Osteomusculares. Foram investigadas 19 variáveis independentes, divididas em quatro categorias: características sociodemográficas, comportamentais, ocupacionais e de saúde. Foi realizada análise univariada e, na sequência, regressão múltipla de Poisson com variância robusta. As variáveis independentes foram inseridas em blocos com critério backward stepwise, considerando o valor para estatística de Wald igual a 0,20 . As medidas de efeito foram expressas em aumento relativo (AR) no valor médio, sendo os dados analisados para um nível de significância de $5 \%$.

RESULTADOS: A prevalência estimada de sintomas osteomusculares nos últimos 12 meses foi de $90 \%$ (intervalo de confiança - IC95\% 87-93). No modelo final da análise de regressão, as variáveis sexo feminino (AR = 14,75\%), índice de capacidade para o trabalho baixo (AR = 100,02\%) e moderado ( $\mathrm{AR}=64,06 \%)$, uso de medicamentos ( $\mathrm{AR}=48,06 \%$ ) e circunferência da cintura em risco $(\mathrm{AR}=15,59 \%)$ tiveram associação significativa com o aumento da média de sintomas; já a escolaridade com ensino técnico atuou como fator de proteção, reduzindo a média em 36,46\%.

CONCLUSÕES: A alta prevalência de sintomas osteomusculares encontrada e os fatores associados indicam a necessidade de propor ações e cuidados específicos para essa população, como tratamento imediato dos sintomas e mudanças na organização e no ambiente laboral, a fim de alcançar equilíbrio e harmonia nas exigências do trabalho sentado prolongado e evitar o impacto dessa condição na saúde pública.

DESCRITORES: Saúde do Trabalhador. Medidas de Associação, Exposição, Risco ou Desfecho. Transtornos Traumáticos Cumulativos. Postura. Estilo de Vida Sedentário. 


\section{INTRODUÇÃO}

A saúde do trabalhador é uma área da saúde pública que tem como objeto de estudo e intervenção as relações entre trabalho e saúde, com dimensões sociais, políticas e técnicas indissociáveis. No âmbito do Sistema Único de Saúde (SUS), a incorporação dessa área não ocorreu de modo linear, mas demandou a superação de obstáculos, formação multiprofissional, esforços na articulação entre suas instâncias e apoios interinstitucionais - que obtiveram avanços, porém ainda apresentam muitos desafios ${ }^{1}$.

A efetiva prevenção dos agravos à saúde do trabalhador demanda transformações aliadas ao apoio e à proteção social. Destaca-se a alta prevalência de distúrbios osteomusculares relacionados ao trabalho (DORT), sendo premente o reconhecimento dos fatores a eles associados e de seus determinantes ${ }^{2}$.

Além disso, existe um crescente interesse em conhecer os efeitos que a postura sentada por tempo prolongado causa à saúde dos indivíduos ${ }^{3-6}$. Essa posição é cada vez mais frequente nos postos de trabalho, o que vem estimulando pesquisadores ${ }^{7}$ a avaliar, quantitativamente, a aceitabilidade, a viabilidade e as percepções, pelos trabalhadores de escritório, do uso de estações de trabalho sit-stand, que permitem a alternância entre ficar sentado e em pé durante o expediente.

Ademais, há maior conscientização e preocupação em distinguir os efeitos da inatividade física e do estilo de vida sedentário, pois um indivíduo pode ser classificado como ativo segundo a definição padronizada pela Organização Mundial da Saúde $(\mathrm{OMS})^{8}$ e, ao mesmo tempo, ter um estilo de vida sedentário, que é o caso de quem permanece muito tempo sentado por dia9.

Visto que os DORT constituem problema de dimensão epidêmica ${ }^{10-12}$ em diversas categorias profissionais, com grande impacto social, econômico e de saúde, principalmente nas atividades administrativas ou de escritório, nas quais os trabalhadores permanecem sentados por longos períodos, em condições que requerem mais estudos e investigações, constata-se a relevância de identificar os principais fatores associados aos sintomas osteomusculares (SO) desses trabalhadores, possibilitando uma abordagem preventiva e intervencionista mais eficiente. Dessa forma, esta pesquisa tem como objetivos estimar a prevalência de $\mathrm{SO}$ e analisar os fatores a eles associados em profissionais de setores administrativos que trabalham predominantemente na postura sentada.

\section{MÉTODOS}

Trata-se de estudo transversal, realizado em órgão da administração pública federal de dois municípios da região Sul do Brasil, com trabalhadores adultos de setores administrativos que atuam, predominantemente, na postura sentada.

Os elegíveis para participação foram pessoas: a) com idade entre 18 e 59 anos, inclusive; b) que tivessem cursado, no mínimo, até o quinto ano do ensino fundamental; c) que trabalhassem na instituição há no mínimo seis meses; e d) que atuassem em setores administrativos na postura sentada há pelo menos seis meses.

Foi considerada postura predominantemente sentada durante o expediente, aquela em que o profissional permanece mais que $50 \%$ de sua carga horária diária de trabalho nessa postura - condição autorrelatada pelo participante. $\mathrm{O}$ autorrelato sobre o tempo sentado é utilizado em muitos estudos e tem-se mostrado confiável ${ }^{5,6}$.

Foram excluídos do estudo aqueles: a) em afastamento do trabalho, licença ou férias até um mês antes ou durante a coleta de dados; b) gestantes; c) que possuíssem SO em consequência de doenças neurológicas, congênitas, reumáticas ou neoplásicas; d) que apresentassem 
deformidade limitante para a realização dos testes físicos; e) com deficiência vocal e/ou auditiva; e f) que tivessem sofrido queda ou trauma nos últimos três meses.

Para o cálculo amostral foi considerada prevalência média de DORT igual a 60\%, nível de significância $\alpha=0,05$, erro de estimativa $d=0,05$ e acréscimo de $15 \%$ para possíveis perdas, alcançando-se uma amostra de 434 trabalhadores. A amostragem foi feita por conveniência com os trabalhadores dos dois municípios previamente selecionados.

\section{Instrumentos de Coleta de Dados}

Para coleta das informações sobre as variáveis investigáveis (independentes), foi desenvolvido um questionário estruturado a fim de obter dados sociodemográficos, variáveis comportamentais, fatores do trabalho e características de saúde com possíveis associações aos DORT. Esse questionário teve a validade de seu conteúdo analisada por uma equipe de sete doutores antes do início da pesquisa, obtendo-se um índice de validade de conteúdo de 0,98.

Deste modo, o questionário foi constituído por 19 variáveis independentes, separadas em quatro classes: 1) sociodemográficas (sexo, idade e escolaridade); 2) comportamentais (atividade física, tabagismo, tempo na postura sentada além do trabalho e uso do computador após o trabalho); 3) ocupacionais (horas extras, tempo diário sentado no trabalho e tempo de trabalho predominantemente na postura sentada - considerando ocupações anteriores, frequência do uso do computador, condições ergonômicas do posto de trabalho e índice de capacidade para o trabalho - ICT); e 4) saúde (uso de medicamento para dor/desconforto osteomuscular nos últimos 12 meses, circunferência da cintura, flexibilidade da cadeia muscular posterior, força muscular da porção inferior dos abdominais, encurtamento dos flexores do quadril e resistência dos músculos abdominais).

A variável dependente foi a prevalência de SO nos últimos 12 meses, investigada com o Questionário Nórdico de Sintomas Osteomusculares (QNSO), em sua versão geral, já validado no Brasil ${ }^{13}$. A interpretação desse desfecho foi realizada de forma numérica, preservando os relatos dos trabalhadores, que poderiam citar nenhum (zero) ou até nove SO.

Para a identificação relacionada à prática de atividade física foi utilizada a classificação da OMS, que recomenda para adultos 150 minutos de atividade física aeróbica de intensidade moderada por semana, ou pelo menos 75 minutos em intensidade vigorosa, sendo aceita também uma combinação equivalente de atividades de intensidade moderada e vigorosa ${ }^{8}$.

A avaliação das condições ergonômicas dos postos de trabalho informatizados foi realizada com o checklist de Couto, versão 2014 $4^{14}$. Já para avaliar a capacidade laboral, foi utilizado o ICT em versão traduzida e adaptada para o Brasil ${ }^{15}$.

Optou-se, neste estudo, por utilizar a terminologia "circunferência da cintura" (CC), com base em estudo de revisão sistemática ${ }^{16}$, o ponto médio entre a última costela e a crista ilíaca como sítio anatômico de medida e pontos de corte segundo a International Diabetes Federation ${ }^{17}$ - o padrão mais utilizado internacionalmente e adotado pela Sociedade Brasileira de Cardiologia.

A força dos músculos abdominais inferiores foi identificada de acordo com o procedimento sugerido por Kendall et al. ${ }^{18}$ Já para avaliar o encurtamento dos músculos flexores do quadril, foi utilizado o teste de Thomas, conforme propõe Magee $^{19}$. A flexibilidade dos músculos da região posterior do tronco e dos membros inferiores, denominada também de cadeia muscular posterior, foi medida pelo teste de sentar e alcançar com banco de Wells, por ser um método muito utilizado em pesquisa ${ }^{20}$.

A resistência ou endurance dos músculos abdominais foi avaliada com o teste do abdominal de um minuto, que consiste em realizar o número máximo de abdominais possível nesse intervalo de tempo. Os valores normativos para esse teste consideram o sexo e a idade da pessoa e classificam a resistência em excelente, acima da média, média, abaixo da média ou fraca ${ }^{21}$. 


\section{Procedimentos de Coleta e Análise dos Dados}

A coleta de dados foi realizada por uma equipe de cinco examinadores, que receberam um treinamento de 20 horas sobre aspectos relacionados às técnicas de entrevista, aos métodos e aos procedimentos dos testes e instrumentos, objetivando padronização e calibração. Foi criado um grupo em aplicativo de comunicação no celular para resolução de dúvidas imediatas, além de serem feitas reuniões presenciais quinzenais. As entrevistas e os testes físicos foram realizados no próprio local de trabalho, porém em sala privada - devido ao uso de colchonete e banco de Wells e para preservar a privacidade do participante -, com duração média de 35 minutos.

\section{Análises Estatísticas}

A apresentação dos resultados foi realizada mediante uma abordagem descritiva e outra analítica. Na abordagem descritiva, apresentam-se a distribuição de frequência absoluta e relativa e medidas de tendência central para descrever as variáveis quantitativas. Quanto à abordagem analítica, foi obtido um intervalo com 95\% de confiança (IC95\%) para estimar a prevalência de SO, e foram feitas análises univariadas na comparação de todas as variáveis independentes com a dependente. Já na definição dos grupos de comparação segundo as categorias da variável ou na categorização de variáveis numéricas, para amostras grandes (n > 30) foram utilizados os testes paramétricos $t$ de Student (para dois grupos independentes) e Anova (para três ou mais grupos independentes). Em todos os casos, intervalos com 95\% de confiança foram obtidos para as médias. Para as variáveis numéricas, sem categorização, foi empregado o teste de correlação linear de Pearson.

Nas amostras pequenas e no caso de normalidade não satisfeita, verificada por meio do teste Shapiro-Wilk, foi empregado o teste não paramétrico Kruskal-Wallis (para três ou mais grupos independentes). A homocedasticidade entre grupos foi verificada pelo teste de Levene (paramétrico) ou pelo teste de Fligner-Killer (não paramétrico). Optou-se pela análise de regressão de Poisson com variância robusta, uma vez que é referência para análise de dados de contagem e pelo fato de o odds ratio ter a tendência de superestimar a razão de prevalência quando o desfecho é comum ou elevado ${ }^{22}$.

Utilizou-se um modelo no qual as variáveis independentes foram inseridas em blocos na seguinte ordem: dados sociodemográficos, comportamentais, ocupacionais e de saúde. Foram incluídas as variáveis com significância menor que $0,20(p<0,20)$ nas análises univariadas. A seleção das variáveis no modelo foi realizada com o critério backward stepwise, considerando-se também um valor igual a 0,20 para a estatística de Wald na manutenção das variáveis durante a análise ajustada nível a nível, a fim de controlar potenciais fatores de confusão. As medidas de efeito foram expressas em aumento relativo (AR) na média.

As análises finais foram consideradas em um nível de significância de $5 \%(\alpha=0,05)$. Os testes foram realizados nos programas estatísticos R versão 3.4.2 e Statistical Package for the Social Sciences (SPSS) versão 22.0. A pesquisa foi aprovada em 21 de novembro de 2017 pelo Comitê de Ética em Pesquisa, envolvendo Seres Humanos da Escola de Enfermagem de Ribeirão Preto da Universidade de São Paulo sob o protocolo CAAE 74543517.8.0000.5393, onde todos os participantes assinaram termo de consentimento livre e esclarecido.

\section{RESULTADOS}

Participaram da pesquisa 451 trabalhadores, com idade média de 44,4 anos, sendo a maioria do sexo feminino (54,5\%), com ensino superior ou pós-graduação (81,2\%), não fumante $(84,3 \%)$ e praticante de atividade física regularmente (53,9\%). Permaneciam sentados em média 6,51 horas no trabalho e mais 3,12 horas durante o tempo de lazer/descanso. As demais características comportamentais, ocupacionais e de saúde encontram-se na Tabela 1, e as variáveis numéricas na Tabela 2. Não houve significância estatística em qualquer análise de correlação das variáveis numéricas com o número de SO nos últimos 12 meses relatado pelos trabalhadores (Tabela 2). 
Tabela 1. Distribuição dos participantes segundo características sociodemográficas, comportamentais, ocupacionais e de saúde $(n=451)$ em municípios da região Sul do Brasil.

\begin{tabular}{|c|c|c|c|}
\hline & & Frequência (n) & Porcentagem (\%) \\
\hline \multirow{2}{*}{ Sexo } & Feminino & 246 & 54,5 \\
\hline & Masculino & 205 & 45,5 \\
\hline \multirow{4}{*}{ Escolaridade } & Ensino médio & 62 & 13,7 \\
\hline & Ensino técnico & 23 & 5,1 \\
\hline & Ensino superior & 260 & 57,6 \\
\hline & Pós-graduação & 106 & 23,6 \\
\hline \multirow{4}{*}{ Tabagismo } & Fumante diário & 17 & 3,8 \\
\hline & Fumante ocasional & 9 & 2,0 \\
\hline & Ex-fumante & 45 & 10,0 \\
\hline & Não fumante & 380 & 84,3 \\
\hline \multirow{2}{*}{ Prática de atividade física } & Sim & 243 & 53,9 \\
\hline & Não & 208 & 46,1 \\
\hline \multirow{2}{*}{$\begin{array}{l}\text { Uso do computador fora do } \\
\text { trabalho }\end{array}$} & Sim & 179 & 39,7 \\
\hline & Não & 272 & 60,3 \\
\hline \multirow{2}{*}{ Hora extra } & Sim & 34 & 7,5 \\
\hline & Não & 417 & 92,5 \\
\hline \multirow{3}{*}{$\begin{array}{l}\text { Frequência do uso do computador } \\
\text { no trabalho }\end{array}$} & Menos de $50 \%$ do tempo & 13 & 2,9 \\
\hline & Entre $50 \%$ e $70 \%$ do tempo & 35 & 7,8 \\
\hline & Acima de $70 \%$ do tempo & 403 & 89,4 \\
\hline \multirow{4}{*}{ ICT } & Baixo & 6 & 1,3 \\
\hline & Moderado & 79 & 17,5 \\
\hline & Bom & 204 & 45,2 \\
\hline & Ótimo & 162 & 35,9 \\
\hline \multirow{2}{*}{$\begin{array}{l}\text { Uso de medicamento nos últimos } \\
12 \text { meses }\end{array}$} & Sim & 244 & 54,1 \\
\hline & Não & 207 & 45,9 \\
\hline \multirow{3}{*}{ Circunferência da cintura } & Menor risco & 202 & 44,8 \\
\hline & Risco aumentado & 147 & 32,6 \\
\hline & $\begin{array}{l}\text { Risco substancialmente } \\
\text { aumentado }\end{array}$ & 102 & 22,6 \\
\hline \multirow{5}{*}{ Flexibilidade (banco de Wells) } & Excelente & 41 & 9,1 \\
\hline & Acima da média & 63 & 14,0 \\
\hline & Média & 69 & 15,3 \\
\hline & Abaixo da média & 81 & 18,0 \\
\hline & Ruim & 197 & 43,7 \\
\hline \multirow{3}{*}{$\begin{array}{l}\text { Força muscular dos abdominais } \\
\text { inferiores }\end{array}$} & Regular & 170 & 37,7 \\
\hline & Boa & 235 & 52,1 \\
\hline & Normal & 46 & 10,2 \\
\hline \multirow{5}{*}{ Resistência dos abdominais } & Excelente & 94 & 20,8 \\
\hline & Acima da média & 81 & 18,0 \\
\hline & Média & 74 & 16,4 \\
\hline & Abaixo da média & 66 & 14,6 \\
\hline & Fraca & 136 & 30,2 \\
\hline \multirow{2}{*}{$\begin{array}{l}\text { Encurtamento dos flexores do } \\
\text { quadril }\end{array}$} & Sim & 80 & 17,7 \\
\hline & Não & 371 & 82,3 \\
\hline
\end{tabular}

ICT: índice de capacidade para o trabalho.

A prevalência estimada de SO nos últimos 12 meses foi de 90\% (IC95\% 87-93), com média de 3,34 sintomas por participante. Na análise dos $\mathrm{SO}$ em relação às variáveis sociodemográficas, observou-se maior frequência entre as mulheres, com média de 
3,68 SO nos últimos 12 meses, enquanto os homens obtiveram uma média de 2,94, com $\mathrm{p}<0,001$. Em relação à escolaridade, aqueles com ensino técnico apresentaram a menor mediana de $\mathrm{SO}$ quando comparados aos participantes com outros níveis de escolaridade, com $\mathrm{p}=0,027$ (Tabela 3).

Quanto às variáveis comportamentais, os inativos fisicamente apresentaram maior média de $\mathrm{SO}(\bar{x}=3,62)$ que os que praticavam atividade física $(\bar{x}=3,11)$, com $\mathrm{p}=0,014$. Já na análise do hábito de fumar e do uso do computador fora do trabalho, não foram observadas diferenças significativas entre os grupos (Tabela 3).

Quanto à relação dos SO com as variáveis ocupacionais, ficou demonstrado que os trabalhadores com ICT baixo também tiveram mais SO, com mediana de 5,5, enquanto os que obtiveram ótimo ICT apresentaram mediana de 2 , com $\mathrm{p}<0,001$ (Tabela 4).

Tabela 2. Dados descritivos e análises de correlação das variáveis numéricas com a quantidade de sintomas osteomusculares nos últimos 12 meses $(n=451)$ em municípios da região Sul do Brasil.

\begin{tabular}{lccccc}
\hline & Idade (anos) & $\begin{array}{c}\text { Tempo } \\
\text { sentado fora } \\
\text { do trabalho } \\
\text { (horas/dia) }\end{array}$ & $\begin{array}{c}\text { Tempo sentado } \\
\text { no trabalho } \\
\text { (horas/dia) }\end{array}$ & $\begin{array}{c}\text { Tempo em } \\
\text { trabalho sentado } \\
\text { (em anos) }\end{array}$ & $\begin{array}{c}\text { Média final do } \\
\text { checklist de } \\
\text { Couto (em \%) }\end{array}$ \\
\hline $\begin{array}{l}\text { Dados descritivos: } \\
\text { valor médio e } \\
\text { desvio-padrão (DP) }\end{array}$ & $\begin{array}{c}44,4 \\
(\mathrm{DP}=10,6)\end{array}$ & $\begin{array}{c}3,12 \\
(\mathrm{DP}=1,55)\end{array}$ & $\begin{array}{c}6,51 \\
(\mathrm{DP}=0,96)\end{array}$ & $\begin{array}{c}20,29 \\
(\mathrm{DP}=10,88)\end{array}$ & $\begin{array}{c}88,9 \% \\
(\mathrm{DP}=4,37)\end{array}$ \\
$\begin{array}{l}\text { Quantidade de SO } \\
\text { nos últimos 12 meses }\end{array}$ & 0,083 & 0,082 & 0,033 & 0,079 & 0,068 \\
$\begin{array}{l}\text { Coeficiente de } \\
\text { correlação }\end{array}$ & 0,077 & 0,082 & 0,489 & 0,093 & 0,152 \\
\hline \begin{tabular}{l} 
Significância bilateral \\
\hline
\end{tabular}
\end{tabular}

SO: sintomas osteomusculares.

a Teste de correlação de Pearson.

Tabela 3. Dados sociodemográficos e comportamentais em relação com os sintomas osteomusculares nos últimos 12 meses $(\mathrm{n}=451)$ em municípios da região Sul do Brasil.

\begin{tabular}{|c|c|c|c|c|c|c|c|c|}
\hline & & Média & Desvio-padrão & IC95\% & Mediana & Mínimo & Máximo & $\mathbf{p}$ \\
\hline \multirow{2}{*}{ Sexo } & Feminino & 3,68 & 2,168 & $3,41-3,96$ & 4,00 & 0 & 9 & $<0,001$ \\
\hline & Masculino & 2,94 & 2,154 & $2,64-3,23$ & 3,00 & 0 & 9 & \\
\hline \multicolumn{9}{|l|}{ Teste $t$ de Student } \\
\hline \multirow{4}{*}{ Escolaridade } & Ensino médio & - & - & - & 3,00 & 0 & 9 & 0,027 \\
\hline & Ensino técnico & - & - & - & 2,00 & 0 & 6 & \\
\hline & Ensino superior & - & - & - & 3,00 & 0 & 9 & \\
\hline & Pós-graduação & - & - & - & 3,00 & 0 & 9 & \\
\hline \multicolumn{9}{|l|}{ Teste Kruskal-Wallis } \\
\hline \multirow{4}{*}{ Tabagismo } & Fumante diário & - & - & - & 5,00 & 0 & 8 & 0,397 \\
\hline & Fumante ocasional & - & - & - & 4,00 & 1 & 6 & \\
\hline & Ex-fumante & - & - & - & 3,00 & 0 & 7 & \\
\hline & Não fumante & - & - & - & 3,00 & 0 & 9 & \\
\hline \multicolumn{9}{|l|}{ Teste Kruskal-Wallis } \\
\hline \multirow{2}{*}{ Prática de atividade física } & $\operatorname{Sim}$ & 3,11 & 2,078 & $2,84-3,37$ & 3,00 & 0 & 9 & 0,014 \\
\hline & Não & 3,62 & 2,289 & $3,31-3,93$ & 4,00 & 0 & 9 & \\
\hline \multicolumn{9}{|l|}{ Teste $t$ de Student } \\
\hline \multirow{2}{*}{$\begin{array}{l}\text { Uso do computador fora } \\
\text { do trabalho }\end{array}$} & Sim & 3,23 & 2,173 & $2,91-3,56$ & 3,00 & 0 & 9 & 0,392 \\
\hline & Não & 3,42 & 2,204 & $3,15-3,68$ & 3,00 & 0 & 9 & \\
\hline
\end{tabular}


As análises univariadas das variáveis de saúde em relação à presença de SO nos últimos 12 meses mostraram que as pessoas que utilizaram medicamentos para dor no último ano obtiveram maior média de SO $(\bar{x}=4,10)$ do que as que não utilizaram $(\bar{x}=2,45)$, com $\mathrm{p}<0,001$ (Tabela 4).

Tabela 4. Dados ocupacionais e de saúde em relação com os sintomas osteomusculares nos últimos 12 meses (n = 451) em municípios da região Sul do Brasil.

\begin{tabular}{ccccccccccc}
\hline & & Média & Desvio-padrão & IC95\% & Mediana & Mínimo & Máximo & p \\
\hline \multirow{2}{*}{ Hora extra } & Sim & 3,15 & 1,893 & $2,49-3,81$ & 3,00 & 0 & 7 & 0,587 \\
& Não & 3,36 & 2,214 & $3,15-3,57$ & 3,00 & 0 & 9 &
\end{tabular}

Teste $t$ de Student

\begin{tabular}{|c|c|c|c|c|c|c|c|c|}
\hline \multirow{3}{*}{$\begin{array}{l}\text { Frequência do uso do } \\
\text { computador no trabalho }\end{array}$} & $\begin{array}{c}\text { Menos de } 50 \% \text { do } \\
\text { tempo }\end{array}$ & - & - & - & 2,00 & 1 & 7 & \multirow[t]{3}{*}{0,614} \\
\hline & $\begin{array}{c}\text { Entre } 50 \% \text { e } 70 \% \text { do } \\
\text { tempo }\end{array}$ & - & - & - & 3,00 & 0 & 8 & \\
\hline & $\begin{array}{c}\text { Acima de } 70 \% \text { do } \\
\text { tempo }\end{array}$ & - & - & - & 3,00 & 0 & 9 & \\
\hline \multicolumn{9}{|l|}{ Teste Kruskal-Wallis } \\
\hline \multirow{4}{*}{ ICT } & Baixo & - & - & - & 5,50 & 2 & 9 & \multirow[t]{4}{*}{$<0,001$} \\
\hline & Moderado & - & - & - & 5,00 & 0 & 9 & \\
\hline & Bom & - & - & - & 3,50 & 0 & 9 & \\
\hline & Ótimo & - & - & - & 2,00 & 0 & 8 & \\
\hline
\end{tabular}

Teste Kruskal-Wallis

\begin{tabular}{|c|c|c|c|c|c|c|c|c|}
\hline \multirow{2}{*}{$\begin{array}{l}\text { Uso de medicamento nos } \\
\text { últimos } 12 \text { meses }\end{array}$} & Sim & 4,10 & 2,031 & $3,85-4,36$ & 4,00 & 0 & 9 & \multirow[t]{2}{*}{$<0,001$} \\
\hline & Não & 2,45 & 2,033 & $2,17-2,73$ & 2,00 & 0 & 9 & \\
\hline \multicolumn{9}{|l|}{ Teste $t$ de Student } \\
\hline \multirow{3}{*}{ Circunferência da cintura } & Menor risco & 3,08 & 2,098 & $2,79-3,37$ & 3,00 & 0 & 9 & \multirow[t]{3}{*}{$<0,001$} \\
\hline & Risco aumentado & 3,12 & 2,225 & $2,75-3,48$ & 3,00 & 0 & 9 & \\
\hline & RSA & 4,20 & 2,125 & $3,78-4,61$ & 4,00 & 0 & 9 & \\
\hline
\end{tabular}

Teste Anova

\begin{tabular}{|c|c|c|c|c|c|c|c|c|}
\hline \multirow{5}{*}{$\begin{array}{l}\text { Flexibilidade (banco de } \\
\text { Wells) }\end{array}$} & Excelente & - & - & - & 3,00 & 0 & 7 & \multirow[t]{5}{*}{0,602} \\
\hline & Acima da média & - & - & - & 3,00 & 0 & 8 & \\
\hline & Média & - & - & - & 3,00 & 0 & 9 & \\
\hline & Abaixo da média & - & - & - & 3,00 & 0 & 9 & \\
\hline & Ruim & - & - & - & 3,00 & 0 & 9 & \\
\hline \multicolumn{9}{|l|}{ Teste Kruskal-Wallis } \\
\hline \multirow{3}{*}{$\begin{array}{l}\text { Força muscular dos } \\
\text { abdominais inferiores }\end{array}$} & Regular & 3,61 & 2,182 & $3,28-3,94$ & 3,50 & 0 & 9 & \multirow[t]{3}{*}{0,134} \\
\hline & Boa & 3,17 & 2,248 & $2,88-3,45$ & 3,00 & 0 & 9 & \\
\hline & Normal & 3,28 & 1,846 & $2,73-3,83$ & 3,00 & 0 & 8 & \\
\hline \multicolumn{9}{|l|}{ Teste Anova } \\
\hline \multirow{5}{*}{$\begin{array}{l}\text { Resistência dos } \\
\text { abdominais }\end{array}$} & Excelente & 2,85 & 2,190 & $2,40-3,30$ & 2,50 & 0 & 9 & \multirow[t]{5}{*}{0,072} \\
\hline & Acima da média & 3,40 & 2,017 & $2,95-3,84$ & 3,00 & 0 & 9 & \\
\hline & Média & 3,46 & 2,121 & $2,97-3,95$ & 3,00 & 0 & 8 & \\
\hline & Abaixo da média & 3,17 & 2,351 & $2,59-3,74$ & 3,00 & 0 & 9 & \\
\hline & Fraca & 3,68 & 2,211 & $3,30-4,05$ & 3,50 & 0 & 9 & \\
\hline \multicolumn{9}{|l|}{ Teste Anova } \\
\hline \multirow{2}{*}{$\begin{array}{l}\text { Encurtamento dos flexores } \\
\text { do quadril }\end{array}$} & Sim & 3,63 & 2,420 & $3,09-4,16$ & 3,00 & 0 & 9 & \multirow[t]{2}{*}{0,245} \\
\hline & Não & 3,28 & 2,137 & $3,06-3,50$ & 3,00 & 0 & 9 & \\
\hline
\end{tabular}

SO: sintomas osteomusculares; ICT: índice de capacidade para o trabalho; RSA: risco substancialmente aumentado; IC95\%: intervalo com 95\% de confiança.

Valores com significância estatística estão apresentados em negrito. 
Os trabalhadores classificados, por meio da medida da circunferência da cintura, na categoria de risco substancialmente aumentado para doenças metabólicas e outras complicações tiveram média de SO mais elevada $(4,20)$ do que os que estavam em menor risco (3,08), com $\mathrm{p}<0,001$. Na análise da flexibilidade, não houve diferença estatisticamente significativa entre os grupos ( $p=0,602)$, e todas as categorias de classificações obtiveram mediana igual a 3 (Tabela 4).

Avaliando a relação com a força muscular dos abdominais inferiores, aqueles com força regular registraram uma média de SO de 3,61 e IC95\% 3,28-3,94. Já no estudo da resistência dos músculos abdominais, avaliada no teste de um minuto, não foi constatada diferença significativa entre os grupos, sendo a média dos trabalhadores com fraca resistência muscular de 3,68 SO. Com valor muito similar, aqueles com encurtamento dos flexores do quadril obtiveram média de SO de $\bar{x}=3,63$ e IC95\% 3,09-4,16, sem diferença significativa entre os grupos (Tabela 4).

A partir dos resultados das análises univariadas, foram selecionadas para o modelo de regressão doze variáveis: idade; sexo; escolaridade; prática de atividade física; tempo sentado fora do trabalho; tempo em trabalhos predominantemente na postura sentada; condições ergonômicas do posto de trabalho; ICT; uso de medicamentos nos últimos 12 meses; circunferência da cintura; força muscular dos abdominais inferiores; e resistência dos abdominais.

Os resultados da análise de regressão múltipla, após todas as etapas por blocos (variáveis sociodemográficas, comportamentais, ocupacionais e de saúde), mostraram cinco fatores associados significativamente à média de SO. A Tabela 5 apresenta todas as variáveis que permaneceram no modelo até o final da análise e seus respectivos valores estimados de AR na média, IC95\% e significância estatística (p-valor).

As mulheres tiveram um AR na média de SO de 14,75\% ( $\mathrm{p}$ = 0,0280) em comparação com os homens. Na análise da escolaridade, os trabalhadores com ensino técnico apresentaram redução de $36,46 \%(\mathrm{p}=0,0112)$ na média de $\mathrm{SO}$ em relação àqueles com ensino médio, demostrando ser este um fator de proteção (Tabela 5).

Os trabalhadores com ICT baixo e moderado apresentaram AR de 100,02\% ( $p=0,0007$ ) e 64,06\% ( $\mathrm{p}<0,0001$ ) na média do número de sintomas, respectivamente, em relação aos com ICT ótimo. Já os participantes que fizeram uso de medicamentos para dores osteomusculares nos últimos 12 meses tiveram AR na média de 48,06\% ( $p$ < 0,0001) quando comparados aos que não utilizaram medicamentos (Tabela 5).

Tabela 5. Modelo de regressão de Poisson com variância robusta em $(n=451)$ municípios da região Sul do Brasil.

\begin{tabular}{lccccccc}
\hline Parâmetros & Estimativa & Erro-padrão & z-valor & p-valor & AR & IC95\% \\
\hline Intercept) & 0,6763 & 0,1090 & 6,2061 & 0,0000 & 1,9667 & 1,0149 \\
Sexo feminino & 0,1376 & 0,0626 & 2,1967 & $\mathbf{0 , 0 2 8 0}$ & 1,1475 & 0,2974 \\
Escolaridade: ensino técnico & $-0,4535$ & 0,1788 & $-2,5361$ & $\mathbf{0 , 0 1 1 2}$ & 0,6354 & 0,4475 & 0,9021 \\
Escolaridade: ensino superior & $-0,1134$ & 0,0851 & $-1,3323$ & 0,1828 & 0,8928 & 0,7556 & 1,0549 \\
Escolaridade: pós-graduação & $-0,0573$ & 0,0999 & $-0,5739$ & 0,5660 & 0,9443 & 0,7764 & 1,1485 \\
ICT baixo & 0,6933 & 0,2050 & 3,3817 & $\mathbf{0 , 0 0 0 7}$ & 2,0002 & 1,3384 & 2,9894 \\
ICT moderado & 0,4950 & 0,0868 & 5,7035 & $\mathbf{0 , 0 0 0 0}$ & 1,6406 & 1,3839 & 1,9448 \\
$\begin{array}{l}\text { Uso de medicamentos nos últimos } \\
\text { 12 meses }\end{array}$ & 0,3924 & 0,0643 & 6,1008 & $\mathbf{0 , 0 0 0 0}$ & 1,4806 & 1,3052 & 1,6795 \\
CC em risco aumentado & $-0,0421$ & 0,0702 & $-0,5996$ & 0,5488 & 0,9588 & 0,8355 & 1,1002 \\
$\begin{array}{l}\text { CC em risco substancialmente } \\
\text { aumentado }\end{array}$ & 0,1449 & 0,0738 & 1,9630 & $\mathbf{0 , 0 4 9 6}$ & 1,1559 & 1,0002 & 1,3358 \\
\hline AR: aum & & & &
\end{tabular}

AR: aumento relativo na média; IC95\%: intervalo com 95\% de confiança; ICT: índice de capacidade para o trabalho; CC: circunferência da cintura. Valores com significância estatística estão apresentados em negrito. 
Por fim, na medida da circunferência da cintura, os participantes com risco substancialmente aumentado para síndrome metabólica e outras complicações apresentaram AR na média de SO de $15,59 \%(\mathrm{p}=0,0496)$ com relação aos trabalhadores com menor risco (Tabela 5).

\section{DISCUSSÃO}

A estimativa da prevalência de SO nos últimos 12 meses foi elevada (90\%). Alguns estudos constataram alta prevalência de distúrbios osteomusculares entre indivíduos que trabalham predominantemente sentados, como os trabalhadores de escritórios $(88,4 \%)^{10}$ e informática $(76 \%)^{11}$.

As dores foram significativamente mais frequentes nas mulheres quando comparadas aos homens, com AR de 14,75\% na média de sintomas. Esse dado é regularmente relatado por autores que estudam SO em trabalhadores. No estudo de Scopel et al. ${ }^{12}$, os homens tiveram menor prevalência de casos sugestivos de DORT, com razão de prevalência estimada de 0,62 (IC95\% 0,47-0,81).

Entre os trabalhadores de escritório que utilizam computador, as mulheres apresentaram mais SO em todas as regiões anatômicas avaliadas, e na análise de associação multivariada obtiveram uma razão de chance ou odds ratio igual a 2,4 ( $\mathrm{p}=0,03)$ para a região da cervical e igual a 2,8 $(\mathrm{p}=0,01)$ para membros superiores (braço, cotovelo, antebraço, punho ou mão) ${ }^{11}$. Algumas possíveis explicações para esses achados seriam a menor massa muscular, em termos absolutos e relativos, na composição física da mulher ${ }^{19}$; alterações no sistema hormonal; e a dupla jornada de trabalho, já que muitas vezes ela é a responsável pelos cuidados familiares.

$\mathrm{Na}$ análise da escolaridade, aqueles que tinham curso técnico apresentaram menos sintomas, servindo isso como um fator de proteção. Parece não haver padrão estabelecido quanto a essa variável. Algumas pesquisas demostraram não haver associação da escolaridade com $\mathrm{SO}^{23,24}$, enquanto outras encontraram uma associação de $\mathrm{SO}$ com menor grau de estudos ${ }^{12,25}$.

Os trabalhadores classificados com ICT baixo apresentaram um AR na média de SO significante em relação aos com ICT ótimo. Iunes et al. ${ }^{26}$ evidenciaram associação entre o ICT e a presença de sintomas em todas as regiões descritas no QNSO.

Walsh et al. ${ }^{27}$ observaram que, dentre os trabalhadores com ICT pobre/baixo, $87 \%$ tinham intensidade da dor entre 7 e 10, enquanto $73 \%$ dos com ICT excelente pontuaram de 0 a 2. No estudo de Martinez e Latorre ${ }^{28}$, todas as dimensões da saúde analisadas, inclusive a avaliação da dor, foram associadas à capacidade para o trabalho, sendo esta tão melhor quanto maior for a qualidade da saúde.

Essa relação parece ocorrer nos dois sentidos, ou seja, o trabalhador que possui um número elevado de SO tende a ter um baixo ICT, e aquele que possui um alto ICT tem menor tendência de apresentar SO relacionados ao trabalho. Manter a capacidade para o trabalho é um desafio para os serviços de saúde e o ICT, juntamente com outras avaliações, fornecem aos profissionais e gestores ferramentas e dados essenciais para acompanhar a saúde do trabalhador, permitindo medidas de prevenção e promoção da saúde no ambiente laboral.

Analisando as variáveis de saúde, houve diferença estatisticamente significativa entre os que utilizaram medicamento para dores osteomusculares no último ano e os que não utilizaram. Na análise de regressão observou-se associação significativa, expressando um AR de 48,06\% na média de sintomas. Esse dado pode ser facilmente compreendido, pois a pessoa com dor é mais propensa a utilizar medicamentos. No estudo de Souza et al. ${ }^{29}$, 
64,51\% dos trabalhadores de um hospital público fizeram uso de medicamento para dor na semana anterior à pesquisa.

A associação da circunferência da cintura dos trabalhadores classificados em risco substancialmente aumentado (indicador de obesidade central ou abdominal) com a média de SO manteve-se estatisticamente significativa na análise univariada até o modelo final da regressão de Poisson, com AR de 15,59\%. Magnago et al. ${ }^{25}$ constataram que quase metade dos trabalhadores de enfermagem de um grande hospital universitário do Rio Grande do Sul estava acima do peso ideal, e esse dado foi associado significativamente aos relatos de dor em articulações e coluna lombar.

Para uma possível compreensão dessa associação, vale citar o efeito da sobrecarga nas articulações que ocorre nas pessoas com sobrepeso e obesidade. O estresse e a pressão excessiva causam desgastes nas estruturas e nos tecidos envolvidos (cartilagens, ligamentos, tendões, músculos, entre outros), predispondo à degeneração e à dor ${ }^{30}$.

Alguns pontos relevantes e limitações desta pesquisa merecem destaque, a começar pela apresentação e análise dos dados sobre SO na perspectiva numérica, que considera a influência de cada sintoma relatado e mantém a coerência com o modelo matemático da regressão de Poisson. Outra observação é a apresentação clínica, que pode se mostrar mais relevante e pertinente ao profissional da saúde, visto que em intervenções preventivas e/ou reabilitativas é importante identificar os fatores associados capazes de aumentar o número de SO nos trabalhadores, sem se restringir a cada região corporal do QNSO.

Além das limitações típicas de estudos transversais, como a impossibilidade de estabelecer causalidade, vale citar a questão do autorrelato do tempo sentado, embora a literatura já demonstre certa confiabilidade. Não se observou viés do voluntário sadio, haja vista a alta prevalência de SO, porém deve-se considerar o viés do trabalhador saudável, tendo em conta a exclusão dos trabalhadores afastados por motivos de doença.

Este estudo contribui para o avanço do conhecimento sobre a saúde dos trabalhadores que, independentemente de praticarem atividade física, podem ser classificados num comportamento/estilo de vida sedentário, pois a natureza de sua ocupação profissional exige a postura sentada por longos períodos, diariamente ${ }^{9}$, de modo que ficam submetidos a esse fator de risco, considerado constructo distinto de inatividade física e de crescente interesse na saúde pública.

Portanto, as variáveis sexo feminino, ICT baixo e moderado, uso de medicamentos nos últimos 12 meses e circunferência da cintura acima do recomendável foram estatisticamente associadas ao aumento de SO, sendo a variável ICT a de maior poder de associação. Constatou-se ainda alta prevalência de SO nesses trabalhadores. Isto posto, intervenções para diminuir os SO irão repercutir positivamente na capacidade para o trabalho, o que, por sua vez, tende a diminuir a presença de sintomas.

Assim sendo, uma medida imediata a se adotar é a reabilitação dos trabalhadores com SO. Sabe-se que sintomas iniciais, se negligenciados, podem evoluir para distúrbios incapacitantes, tornando necessário o recurso a afastamentos e auxílios governamentais - o que aumenta um grande problema de saúde pública no Brasil, com repercussões nas dimensões sociais e econômicas.

Além disso, algumas ações peculiares são propostas: adaptações físicas no local de trabalho e ajustes/mudanças na organização das atividades para que os trabalhadores possam ficar em pé por alguns minutos a cada hora e tenham maior gasto energético; orientações e esclarecimentos, com profissionais da saúde especializados, sobre comportamentos de risco e hábitos saudáveis no ambiente laboral; e realização de exercícios específicos para o sistema osteomuscular, que estimulem a circulação sanguínea e desenvolvam características físicas individuais comprovadamente protetoras para quem fica sentado por longos períodos. 


\section{REFERÊNCIAS}

1. Gomez CM, Vasconcellos LCF, Machado JMH. A brief history of worker's health in Brazil's Unified Health System: progress and challenges. Ciênc Saúde Colet. 2018;23(6):1963-70. https://doi.org/10.1590/1413-81232018236.04922018

2. Jackson JM Filho, Pina JA, Vilela RGA, Souza KR. Desafios para a intervenção em saúde do trabalhador. Rev Bras Saúde Ocup. 2018;43(Supl 1):e13s. https://doi.org/10.1590/2317-6369ap0141218

3. Campbell PT, Patel AV, Newton CC, Jacobs EJ, Gapstur SM. Associations of recreational physical activity and leisure time spent sitting with colorectal cancer survival. J Clin Oncol. 2013;31(7):876-85. https://doi.org/10.1200/JCO.2012.45.9735

4. Chomistek AK, Manson JE, Stefanick ML, Lu B, Sands-Lincoln M, Going SB, et al. Relationship of sedentary behavior and physical activity to incident cardiovascular disease: results from the Women's Health Initiative. J Am Coll Cardiol. 2013;61(23):2346-54. https://doi.org/10.1016/j.jacc.2013.03.031

5. George ES, Rosenkranz RR, Kolt GS. Chronic disease and sitting time in middle-aged Australian males: findings from the 45 and Up Study. Int J Behav Nutr Phys Act. 2013;10:20. https://doi.org/10.1186/1479-5868-10-20

6. van der Ploeg HP, Chey T, Korda RJ, Banks E, Bauman A. Sitting time and all-cause mortality risk in 222497 Australian adults. Arch Intern Med. 2012;172(6):494-500. https://doi.org/10.1001/archinternmed.2011.2174

7. Chau JY, Daley M, Srinivasan A, Dunn S, Bauman AE, van der Ploeg HP. Desk-based workers' perspectives on using sit-stand workstations: a qualitative analysis of the Stand@Work study. BMC Public Health. 2014;14:752. https://doi.org/10.1186/1471-2458-14-752

8. World Health Organization. Global recommendations on physical activity for health. Geneva: World Health Organization; 2010 [citado 27 nov 2020]. Disponível em: http://apps.who.int/iris/bitstream/10665/44399/1/ 9789241599979_eng.pdf

9. Pate RR, O'neill JR, Lobelo F. The evolving definition of "sedentary". Exerc Sport Sci Rev. 2008;36(4):173-8. https://doi.org/10.1097/JES.0b013e3181877d1a

10. Mohammadipour F, Pourranjbar M, Naderi S, Rafie F. Work-related musculoskeletal disorders in Iranian office workers: prevalence and risk factors. J Med Life. 2018;11(4):328-33. https://doi.org/10.25122/jml-2018-0054

11. Elshaer N. Prevalence and associated factors related to arm, neck and shoulder complaints in a selected sample of computer office workers. J Egypt Public Health Assoc. 2017;92(4):203-11. https://doi.org/10.21608/EPX.2018.22041

12. Scopel J, Oliveira PAB, Wehrmeister FC. LER/DORT na terceira década da reestruturação bancária: novos fatores associados? Rev Saúde Pública. 2012;46(5):875-85. https://doi.org/10.1590/S0034-89102012000500015

13. Pinheiro FA, Tróccoli BT, Carvalho CV. Validação do Questionário Nórdico de Sintomas Osteomusculares como medida de morbidade. Rev Saúde Pública. 2002;36(3):307-12. https://doi.org/10.1590/S0034-89102002000300008

14. Couto HA. Ergonomia do corpo e do cérebro no trabalho. Belo Horizonte: Ergo; 2014.

15. Tuomi K, Ilmarinen J, Jahkola A, Katajarinne L, Tulkki A. Índice de capacidade para o trabalho. São Carlos: EdUFSCar; 2005.

16. Lima CG, Basile LG, Silveira JQ, Vieira PM, Oliveira MRM. Circunferência da cintura ou abdominal? Uma revisão crítica dos referenciais metodológicos. Rev Simbio-Logias. 2011;4(6):108-31.

17. International Diabetes Federation. The IDF consensus worldwide definition of the metabolic syndrome [Internet]. Brussels: International Diabetes Federation; 2006 [citado 10 jan 2020]. Disponível em: https://www.idf.org/e-library/consensus-statements/60-idfconsensus-worldwidedefinitionof-the-metabolic-syndrome.html

18. Kendall FP, McCreary EK, Provance PG. Músculos: provas e funções com postura e dor. 5a ed. São Paulo: Manole; 2007.

19. Magee DJ. Avaliação musculoesquelética. 5a ed. Barueri: Manole; 2010.

20. Lima POP, Lima AA, Coelho ACS, Lima YL, Almeida GPLA, Bezerra MA, et al. Biomechanical differences in Brazilian jiu-jitsu athletes: the role of combat style. Int J Sports Phys Ther 2017;12(1):67-74 
21. Pollock ML, Wilmore JH. Exercícios na saúde e na doença: avaliação e prescrição para prevenção e reabilitação. 2a ed. Rio de Janeiro: Medsi; 1993.

22. Barros AJD, Hirakata V. Alternatives for logistic regression in cross sectional studies: an empirical comparison of models that directly estimate the prevalence ratio. BMC Med Res Methodol. 2003;3:21. https://doi.org/10.1186/1471-2288-3-21

23. Cardoso RK, Rombaldi AJ, Silva MC. Osteomuscular disorders and associated factors among solid waste collectors of two middle-sized cities from the South of Brazil. Rev Dor. 2014;15(1):13-6. https://doi.org/10.5935/1806-0013.20140004

24. Vitta A, Canocini AA, Conti MHS, Simeão SFAP. Prevalência e fatores associados à dor musculoesquelética em profissionais de atividades sedentárias. Fisioter Mov. 2012;25(2):273-80. https://doi.org/10.1590/S0103-51502012000200004

25. Magnago TSBS, Lisboa MTL, Griep RH, Kirchhof ALC, Camponogara S, Nonnenmacher CQ, Vieira LB. Condições de trabalho, características sociodemográficas e distúrbios musculoesqueléticos em trabalhadores de enfermagem. Acta Paul Enferm. 2010;23(2):187-93. https://doi.org/10.1590/S0103-21002010000200006

26. Iunes DH, Duarte ML, Pereira LV, Nascimento MC, Dionisio VC. Evaluation of musculoskeletal symptoms and of work ability in a higher education institution. Fisioter Mov. 2015;28(2):297-306. https://doi.org/10.1590/0103-5150.028.002.AO10

27. Walsh IAP, Corral S, Franco RN, Canetti EEF, Alem MER, Coury HJCG. Capacidade para o trabalho em indivíduos com lesões músculo-esqueléticas crônicas. Rev Saúde Pública. 2004;38(2):149-56. https://doi.org/10.1590/S0034-89102004000200001

28. Martinez MC, Latorre MRDO. Saúde e capacidade para o trabalho em trabalhadores de área administrativa. Rev Saúde Pública. 2006;40(5):851-8. https://doi.org/10.1590/S0034-89102006000600015

29. Souza DBO, Martins LV, Marcolino AM, Barbosa RI, Tamanini G, Fonseca MCR. Capacidade para o trabalho e sintomas osteomusculares em trabalhadores de um hospital público. Fisioter Pesqui. 2015;22(2):182-90. https://doi.org/10.590/1809-2950/14123722022015

30. Sá K, Baptista AF, Matos MA, Lessa I. Prevalência de dor crônica e fatores associados na população de Salvador, Bahia. Rev Saúde Pública. 2009;43(4):622-30. https://doi.org/10.1590/S0034-89102009005000032

Agradecimentos: À ITAIPU Binacional pelo apoio logístico na realização do doutorado.

Financiamento: Coordenação de Aperfeiçoamento de Pessoal de Nível Superior (Capes - bolsa de doutorado para ARL - Código de Financiamento 001).

Contribuição dos Autores: Concepção e planejamento do estudo: ARL, CBS, CST, MLCCR. Coleta, análise e interpretação dos dados: ARL, CBS, CST, MLCCR, RAR, MJBP. Elaboração e revisão do manuscrito: ARL, CBS, CST, MLCCR, RAR, MJBP. Aprovação da versão final e responsabilidade pública pelo conteúdo do artigo: todos os autores.

Conflito de Interesses: Os autores declaram não haver conflito de interesses. 